\title{
Point-of-care ultrasound defines gastric content and changes the anesthetic management of elective surgical patients who have not followed fasting instructions: a prospective case series L'échographie au chevet détermine le contenu gastrique et modifie la prise en charge anesthésique des patients de chirurgie non urgente qui n'ont pas respecté les consignes de jeûne: une série de cas prospective
}

\author{
Husni Alakkad, MBBS • Richelle Kruisselbrink, MD • Ki Jinn Chin, MD • \\ Ahtsham U. Niazi, MBBS • Sherif Abbas, MD • Vincent W. S. Chan, MD • \\ Anahi Perlas, MD \\ Received: 12 November 2014 / Revised: 7 June 2015 / Accepted: 23 July 2015 / Published online: 4 August 2015 \\ (C) Canadian Anesthesiologists' Society 2015
}

\begin{abstract}
Purpose Pulmonary aspiration of gastric contents is a major cause of anesthesia morbidity and mortality. Pointof-care gastric ultrasound provides information regarding the type and volume of gastric content. The hypothesis of
\end{abstract}

Author contributions Husni Alakkad and Richelle Kruisselbrink were the physician sonographers, and they assisted with data collection. Richelle Kruisselbrink prepared the first draft of the manuscript. Ki Jinn Chin, Ahtsham U. Niazi, and Vincent W.S. Chan assisted the principal investigator with study design and reviewed and revised the manuscript. Sherif Abbas, the study coordinator, was mainly in charge of patient screening, recruitment, and logistics. He summarized the data collected and prepared the Figs and Tables. Anahi Perlas, the principal investigator of the study, was most responsible for study design and protocol development. She obtained Research Ethics Board approval, reviewed and revised the manuscript, and submitted the manuscript for publication. She is the corresponding author.

Presented in part at the University of Toronto Shield's Research Day in May 2013.

Electronic supplementary material The online version of this article (doi:10.1007/s12630-015-0449-1) contains supplementary material, which is available to authorized users.

H. Alakkad, MBBS · R. Kruisselbrink, MD .

K. J. Chin, MD - A. U. Niazi, MBBS - S. Abbas, MD .

V. W. S. Chan, MD · A. Perlas, MD ( $\bowtie)$

Department of Anesthesia, Toronto Western Hospital, University

of Toronto, University Health Network, 399 Bathurst St,

Toronto, ON M5T 2S8, Canada

e-mail: anahi.perlas@uhn.ca

H. Alakkad, MBBS

University of Dammam, Dammam, Saudi Arabia this prospective cohort study was that the addition of pointof-care gastric ultrasound to standard patient assessment results in changes in anesthetic management in at least $30 \%$ of elective surgical patients who do not follow fasting instructions.

Methods Following Research Ethics Board approval and informed consent, elective surgical patients who did not follow fasting instructions were included in this prospective study. Documentation included the type of food ingested, the timing of the ingestion relative to the planned surgical time, and the treating anesthesiologist's management plan based on history alone. Next, an independent anesthesiologist not involved in the medical decision-making performed a focused gastric ultrasound examination. The results of the ultrasound exam were documented in a standardized fashion and made available to the attending anesthesiologist who then confirmed or revised the initial management plan. The treating anesthesiologist's actual (post-test) patient management was documented in a standardized fashion and compared with the initial (pre-test) management plan.

Results Thirty-eight patients were included in this case series. Following point-of-care gastric ultrasound, there was a change in either the timing of anesthesia or the anesthetic technique (or both) in 27 patients (71\%), with a net change towards a lower incidence of surgical delays. Conclusions This prospective case series suggests that a standardized point-of care gastric ultrasound examination informs anesthesiologists' perceived level of aspiration risk and leads to changes in anesthetic management in a 
significant proportion of elective patients who did not follow fasting instructions.

\section{Résumé}

Objectif L'inhalation pulmonaire du contenu gastrique est une cause majeure de morbidité et de mortalité liées à l'anesthésie. L'échographie gastrique au chevet offre des informations concernant le type et le volume du contenu gastrique. L'hypothèse de cette étude de cohorte prospective était de montrer qu'en ajoutant une échographie gastrique au chevet à l'évaluation normale des patients, on modifiait la prise en charge anesthésique d'au moins $30 \%$ des patients de chirurgie non urgente n'ayant pas respecté les consignes de jeûne.

Méthode Après avoir obtenu l'approbation du Comité d'éthique de la recherche et le consentement écrit des patients, des patients devant subir une chirurgie non urgente qui n'avaient pas suivi les consignes de jeûne ont été inclus dans cette étude prospective. Les renseignements colligés comprenaient le type de nourriture ingéré, le moment d'ingestion par rapport à l'heure prévue de la chirurgie, et le plan de prise en charge de l'anesthésiologiste traitant élaboré en fonction du dossier médical seul. Ensuite, un anesthésiologiste indépendant qui n'était pas impliqué dans la prise de décision médicale a réalisé un examen d'échographie gastrique ciblé. Les résultats de l'examen échographique ont été documentés de façon standardisée et mis à disposition de l'anesthésiologiste en charge, qui a par la suite confirmé ou révisé son plan de prise en charge initial. La prise en charge définitive de l'anesthésiologiste traitant (après le test) a été documentée de façon standardisée et comparée au plan de prise en charge initial (avant le test).

Résultats Trente-huit patients ont été inclus dans cette série de cas. Après l'échographie gastrique au chevet, on a observé des changements dans le moment de l'anesthésie ou dans la technique anesthésique (ou les deux) chez 27 patients $(71 \%)$, avec un changement net vers une incidence plus faible de délais chirurgicaux.

Conclusion Cette série de cas prospective suggère qu'un examen échographique standardisé du contenu gastrique au chevet du patient permet à l'anesthésiologiste de mieux évaluer le risque d'inhalation, et entraîne des modifications importantes dans la prise en charge anesthésique des patients de chirurgie non urgente qui n'ont pas respecté les consignes de jeûne.

Pulmonary aspiration of gastric contents is a serious perioperative complication that carries significant morbidity and mortality. ${ }^{1-3}$ A recent national audit by the Royal College of Anaesthetists of the United Kingdom suggests that pulmonary aspiration is the single most common cause of death from anesthesia events and that standard clinical assessment underestimated the level of risk in many cases that resulted in death. ${ }^{4}$ The mainstay of prevention is the application of universal "fasting guidelines" prior to elective surgical procedures to ensure an "empty" stomach at the time of anesthetic induction. ${ }^{5,6}$ Nevertheless, fasting guidelines are applicable only to elective surgical procedures. ${ }^{5}$ In addition, certain physiologic states (e.g., pregnancy) and medical conditions (diabetes, renal or liver dysfunction, and gastroesophageal reflux disease) are associated with delayed gastric emptying. Thus, these individuals may have a "full stomach" despite usually recommended fasting intervals. ${ }^{7-10}$ There are many clinical situations, therefore, in which gastric content and aspiration risk are uncertain or unknown.

Gastric ultrasound provides reliable information about the nature of the content (empty, clear fluid, or solid) and its volume. ${ }^{11-16}$ The aim of this prospective case series was to evaluate if the addition of point-of-care gastric ultrasound to standard patient assessment alters the stratification of aspiration risk and leads to changes in the anesthetic management of elective surgical patients who did not follow fasting instructions.

We hypothesized that changes in anesthetic/surgical timing (proceed as planned, delay, or cancel) occur in at least $30 \%$ of patients. Secondary outcome measures included the incidence of changes in anesthetic technique or airway management strategy and the incidence of perioperative regurgitation, aspiration, and postoperative nausea and vomiting.

\section{Methods}

Initial approval was obtained from the University Health Network Research Ethics Board on April 14, 2011 and renewed annually thereafter. We followed the recommendations of the STROBE statement (STrengthening the Reporting of OBservational studies in Epidemiology) in the conduct and reporting of this prospective case series. ${ }^{17}$ Patients were enrolled in the study from June 2011 to October 2013 at the Toronto Western Hospital. Inclusion criteria were patients aged 18-80 yr, American Society of Anesthesiologists physical status I-III, weight $50-100 \mathrm{~kg}$, height $>150 \mathrm{~cm}$, and presenting for elective surgical or interventional procedures under anesthesia without following fasting instructions. Patients who consumed clear fluids within two hours, a light meal or thick fluids within six hours, or a solid meal within eight hours of the planned surgical procedure were considered for inclusion. Both inpatients and outpatients undergoing any surgical or interventional procedure performed at the Toronto Western Hospital 
(orthopedic, hand surgery, general surgery, neurosurgery, urology, or spine surgery) under regional or general anesthesia were considered for inclusion. Pregnancy was an exclusion criterion since point-of-care gastric sonography has not been fully validated for obstetric patients. In addition, patients with abnormal upper gastrointestinal anatomy (including hiatus hernia, gastric tumours, and previous surgeries of the upper gastrointestinal tract) were excluded as the underlying anomalies could alter the accuracy of the sonographic assessment of gastric volume. The admitting nurse first identified the lack of adherence to fasting instructions during the routine preoperative interview, and both the attending anesthesiologist and one of the study investigators were then informed about the patient at issue. The attending anesthesiologist in charge of patient care interviewed the patient, made an initial assessment, and established a management plan. With the expressed verbal consent from the attending anesthesiologist, the patient was then screened for inclusion in the study and approached by a research associate who explained the study protocol and obtained written informed consent. The fact that participation in the study might result in nonstandard anesthetic management was explicitly discussed.

The following data were recorded in writing at baseline: patient demographics, medical history, details of the latest meal, and pre-test anesthetic management plan as established by the attending anesthesiologist. Two aspects of the management plan were recorded separately: 1) The anesthetic timing was classified as a) proceed with surgery as planned, b) delay the procedure (and by how many hours), and c) cancel the procedure to a different date. 2) The anesthetic technique was documented as a) regional anesthesia and no sedation, b) regional anesthesia and intravenous sedation, c) general anesthesia with a laryngeal mask airway device, d) general anesthesia with endotracheal intubation (ETT), and e) general anesthesia with ETT and rapid sequence induction.

In order to determine the nature and volume of gastric content, the patients underwent a focused bedside gastric ultrasound examination according to a standardized scanning protocol. ${ }^{11,12}$ Ultrasound examinations were conducted by one of two staff anesthesiologists or one of three anesthesiology fellows under staff supervision, with previous experience in gastric ultrasound ranging from four months to five years and a minimum of 50 prior gastric examinations. The investigators performing the ultrasound exams were not directly involved in patient care, and they did not make or suggest any management decision. A curvilinear array low-frequency (2-5 MHz) transducer and a Philips CX50 system (Philips Healthcare, Andover, MA, USA) with image compounding technology were used for the assessments. Patients were first examined in the supine position and then in the right lateral decubitus (RLD) position. A wide "sweep" from the left to the right subcostal margins was performed to localize the antrum and body of the stomach in the epigastric area. The antrum was then imaged in a sagittal plane using the left lobe of the liver and the pancreas and aorta as internal landmarks as previously described (Fig. 1). ${ }^{12}$

Both qualitative (type of content) and quantitative (volume) assessments of gastric content were performed and documented. The findings of the ultrasound exam were summarized and conveyed to the attending anesthesiologist in a standardized fashion according to the following categories: 1) empty stomach (no fluid or solid content visible); 2) low volume of clear fluid $\left(<1.5 \mathrm{~mL} \cdot \mathrm{kg}^{-1}\right)$ consistent with baseline gastric secretions; 3) high volume of clear fluid $\left(>1.5 \mathrm{~mL} \cdot \mathrm{kg}^{-1}\right)$, suggesting greater than baseline gastric secretions; and 4) thick fluid or solid content (Fig. 2). ${ }^{18}$ The stomach was judged to be empty if the antrum appeared flat with a round to ovoid shape and if no content was seen in the lumen in both the supine and RLD positions (grade 0 antrum). ${ }^{15,16}$ The stomach was judged to contain clear fluid if the antrum appeared distended with homogenous hypoechoic or anechoic content (Fig. 1). ${ }^{11,12,16}$ Thick fluid or solid content was deemed to be present if the antrum appeared distended with heterogeneous, hyperechoic, or grossly particular content or if a "frosted glass pattern" was present. ${ }^{11,16}$

If clear fluid was present, the volume of gastric fluid was calculated as a mean of three consecutive readings based on the antral cross-sectional area in the RLD position using a previously validated mathematical model. ${ }^{11,12}$ All images

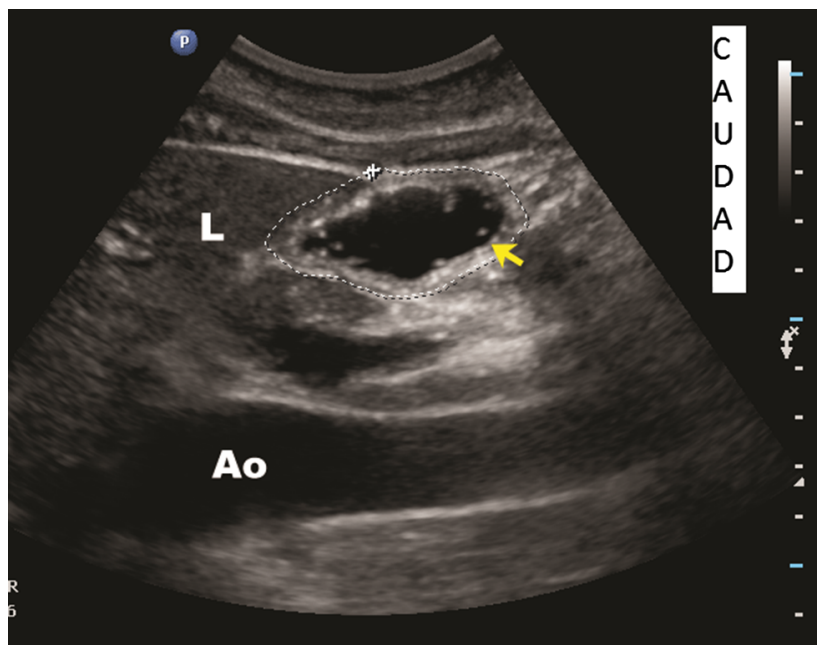

Fig. 1 Sonogram of the gastric antrum obtained in the right lateral decubitus position. The yellow arrow indicates the antrum. The dotted line indicates the measurement of the antral cross-sectional area by the free-tracing method. Ao $=$ aorta; $\mathrm{L}=$ liver 


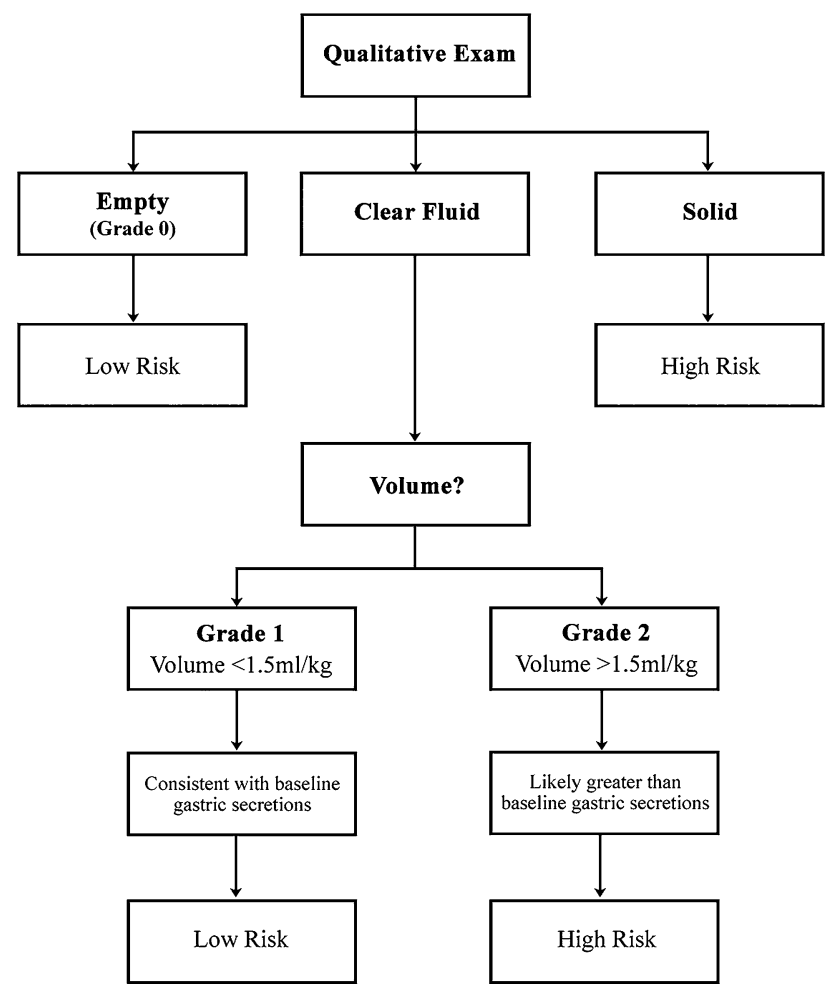

Fig. 2 Schematic representation of the information obtained from point-of-care gastric ultrasound and its implications for aspiration risk assessment. Adapted with permission from: Van de Putte P, Perlas A. Ultrasound assessment of gastric content and volume. Br J Anaesth 2014; 113: $12-22 .{ }^{16}$

were obtained with the stomach at rest between peristaltic contractions.

In addition, the attending anesthesiologist was given the opportunity to be present during the ultrasound examination or to review the resulting images. Furthermore, the attending was afforded the option to request a second ultrasound assessment at a later time if a re-assessment was considered clinically indicated.

Accordingly, the attending anesthesiologist either confirmed or revised the initial management plan, and the actual post-test anesthetic management was documented. Patients were followed until hospital discharge, and during this time, any episodes of nausea, vomiting, regurgitation, or aspiration were recorded.

\section{Sample size calculation and statistical analysis}

Sample size was calculated based on one-sample paired proportions (McNemar's test). We tested the null hypothesis that the addition of a bedside ultrasound assessment of gastric content and volume would not result in any changes in anesthetic management compared with clinical assessment based on patient history. For a hypothesized incidence of changes in anesthetic timing in at least $30 \%$ of the cases, for a twosided McNemar's test, we estimated that a minimum of 35 patients would be required to test the null hypothesis with a type 1 error of $5 \%$ and a power of $80 \%$, assuming a $5 \%$ dropout rate. We used SAS ${ }^{\circledR}$ version 9.3 software (SAS Institute, Cary, NC, USA) for analysis. Data are presented as mean (SD) for continuous variables and count (\%) for categorical variables. McNemar's test (or Bowker's test of symmetry whenever appropriate) was used to test the differences in the anesthetic management plan before vs after the ultrasound examination. Changes in anesthetic timing (proceed as planned, delay, or cancel) and anesthetic technique were recorded and reported separately. All reported $P$ values are two sided.

\section{Results}

Forty-one patients (30 male and 11 female) were screened for possible inclusion during June 2011 to October 2013 (Fig. 3). Screening and enrolment were not consecutive as they were limited by the availability of investigator/sonographers experienced in gastric

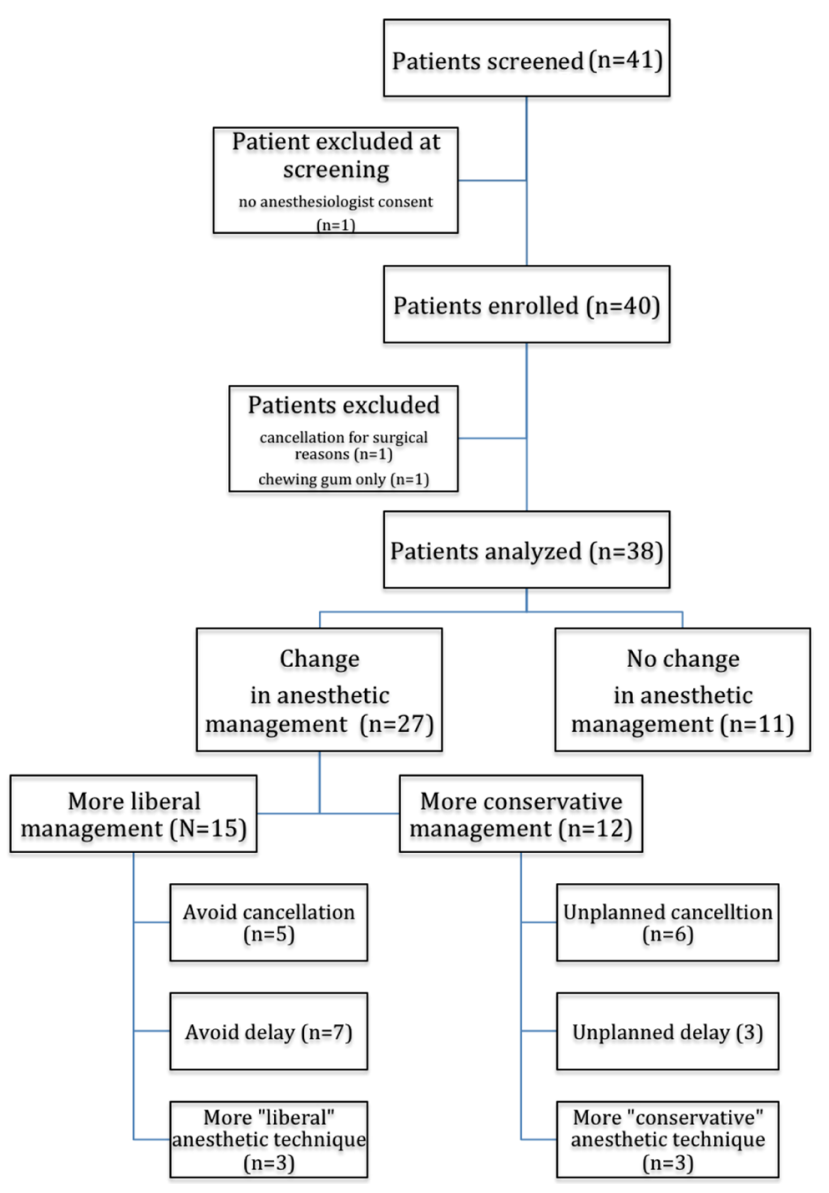

Fig. 3 Patient flow and results 
ultrasound. One patient screened as a possible candidate was not invited to participate due to the lack of consent by the attending anesthesiologist. The remaining 40 patients were referred to the study and all provided informed consent. Two patients were excluded after informed consent. One patient was excluded as he had chewed gum but had otherwise complied with fasting instructions. A second patient was excluded after consent due to a surgical cancellation for unrelated reasons (surgical decision). The remaining 38 patients completed the study assessments and were included in the final analysis (Fig. 3). Patient demographic information is presented in Table 1. Seventeen individual anesthesiologists referred patients to the study, four females and 13 males with a mean (SD) age of 52 (13) yr and 18 (12) yr of previous experience in practice. The referring anesthesiologists were not co-investigators and were not otherwise directly related to the study. The procedures represented all surgical subspecialties at our centre, including general, orthopedic, and plastic surgery; urology; neurosurgery; and interventional radiology (Table 1). Twenty-six patients $(68.4 \%)$ consumed a variety of solid food, usually with an accompanying fluid, while the remaining 12 patients (31.6\%) consumed thick fluids only, usually consisting of particulate fruit juices and/or dairy products (Appendix, available as Electronic Supplementary Material). No patients were referred to the study after consuming clear fluids only. The details of the ingested food, the timing of the ingestion, pre-test anesthetic plan, ultrasound examination findings, and post-test anesthetic

Table 1 Patient demographics, planned anesthetic, and surgery $(n=38)$

\begin{tabular}{ll}
\hline Patient Demographics & \\
Age $(\mathrm{yr})$, mean $(\mathrm{SD})$ & $50(26)$ \\
Sex, $n(\mathrm{M}: \mathrm{F})$ & $28: 10$ \\
Body mass index $\left(\mathrm{kg} \cdot \mathrm{m}^{-2}\right)$, mean $(\mathrm{SD})$ & $26.8(9)$ \\
Gastroesophageal reflux disease, $n(\%)$ & $5(12.8)$ \\
Diabetes mellitus, $n(\%)$ & $10(25.6)$ \\
Planned Anesthesia, $\mathrm{n}(\%)$ & \\
Regional anesthesia & $17(43.6)$ \\
General anesthesia & $16(42.1)$ \\
Regional + general anesthesia & $3(7.7)$ \\
Other & $2(5.1)$ \\
Type of Surgery, $\mathrm{n}(\%)$ & \\
Orthopedics & $15(39.5)$ \\
Plastics & $12(30.8)$ \\
Urology & $1(2.6)$ \\
General surgery & $4(10.25)$ \\
Neurosurgery & $4(10.25)$ \\
Other & $2(5.1)$ \\
\hline
\end{tabular}

$\mathrm{SD}=$ standard deviation management are summarized in the Appendix. Following the result of the first ultrasound examination, there were requests to perform a second examination at a later time in $23(60.5 \%)$ subjects. These cases presented an initial finding consistent with a "full stomach", and the attending anesthesiologist chose to re-assess the situation at a later, clinically appropriate, time before making a final patient care decision. The results of the two ultrasound exams are presented in the Appendix. Following the last ultrasound examination, an empty stomach (grade 0 antrum) was documented in ten cases $(26.3 \%)$, a small volume of clear fluid (grade 1 antrum and $<1.5 \mathrm{~mL} \cdot \mathrm{Kg}^{-1}$ ) similar to that expected in fasted individuals was observed in 14 patients $(36.8 \%)$, and a large amount of clear fluid (grade 2 antrum and $>1.5 \mathrm{~mL} \cdot \mathrm{Kg}^{-1}$ ), thick fluid, or solid content suggesting a full stomach was observed in the remaining 14 patients $(36.8 \%)$ (Appendix).

Following the ultrasound test/s, a change in some aspect of the anesthetic management occurred in 27 cases $(71 \%)$. Of these, 21 subjects $(55.3 \%)$ had a change in the primary outcome, i.e., the timing of anesthesia and surgery (proceed, wait beyond the scheduled time, or cancel) (Table 2). The distribution of this decision was significantly different pre- and post-test $(P=0.008$, Bowker's test of symmetry) (Table 2). Six study subjects (15.8\%) had changes that affected only the anesthetic technique or the airway management strategy. Management did not change after the ultrasound test in the remaining 11 patients (29\%), case numbers $4,5,6,10,13,16,17,19,23,33,34$ (Appendix).

Of the 27 patients with a change in anesthetic management, a more "liberal" strategy consistent with a lower aspiration risk than originally anticipated was used in 15 patients (40\%), and a more "conservative" strategy consistent with a higher than anticipated risk was used in 12 patients (31\%) (Fig. 3). Changes consistent with a lower than anticipated aspiration risk included a) proceeding with surgery rather than cancelling ( $n=5$, case numbers 1,22 , $27,29,35)$, b) proceeding with surgery at the originally scheduled time rather than waiting beyond that time $(n=7$, case numbers $3,12,14,18,20,32,38)$, and c) using a different anesthetic or airway management technique appropriate for a low-risk situation ( $n=3$, case numbers $9,11,26)$. On the other hand, changes consistent with a higher than anticipated aspiration risk included a) cancelling rather than proceeding with a surgical procedure ( $n=6$, case numbers $2,7,21,25,30,31), \mathrm{b}$ ) waiting for a longer period than originally planned $(n=3$, case numbers $24,36,37$ ), and c) changes in anesthetic technique appropriate for a higher risk situation $(n=3$, case numbers $8,15,28)$. There were no adverse events, including no episodes of regurgitation or aspiration in any patient. Seven patients $(18 \%)$ reported nausea and two 
Table 2 Detailed pre-test and post-test anesthetic management plan

\begin{tabular}{llll}
\hline Post-test anesthetic management & \multicolumn{2}{l}{ Pre-test anesthetic plan $(n=38)$} & Proceed $(n=8)$ \\
\cline { 2 - 4 } & Cancel $(n=7)$ & Delay $(n=23)$ & $0(0.0)$ \\
\hline Cancel $(n=8)$ & $2(28.6)$ & $6(26.1)$ & $4(50.0)$ \\
Delay $(n=14)$ & $0(0.0)$ & $10(43.5)$ & $4(50.0)$ \\
Proceed $(n=16)$ & $5(71.4)$ & $7(30.4)$ & 0.008 \\
\hline
\end{tabular}

${ }^{1}$ From Bowker's test of symmetry. Data are expressed as $n(\%)$

patients (5\%) vomited in the immediate perioperative period.

\section{Discussion}

Our data show that a standardized point-of-care ultrasound examination led to changes in management in $27(71 \%)$ patients. Of these, 21 patients (55.3\% of the total cohort) had a revised timing of anesthesia and surgery. This suggests a different perception of aspiration risk following the ultrasound exam compared with baseline standard patient assessment. Interestingly, changes occurred in both directions. An empty stomach was documented in several patients after shorter than anticipated fasting times (e.g., cases $1,6,16$, and 38 ), while others still presented grossly distended stomachs with a significant amount of solid or fluid contents following eight hours of fasting (e.g., cases $2,21,25,28$, and 30). Of the seven cases that would have been cancelled based on standard patient assessment, it is quite significant that five proceeded with surgery at the scheduled time after ultrasound evidence of an empty stomach (cases 1, 22, 27, 29, 35), while findings of solid gastric content confirmed the intention to cancel the procedure in the remaining two cases (cases 5, 34). Conversely, six patients who would have proceeded with surgery based on standard assessment actually had the procedure cancelled after ultrasound confirmed solid gastric content $(n=5$, cases $2,7,21,25,31)$ or gross distention with clear fluid $(n=1$, case 30) despite recommended fasting intervals. Although the net number of actual surgical cancellations was not significantly different than the number planned ( $7 v s 8$, respectively), one might argue that the "right" patients (those with a full stomach) had their surgery cancelled, while those with an empty stomach were able to proceed with surgery safely regardless of the fasting time interval.

A recent national audit by the Royal College of Anaesthetists of the United Kingdom suggests that pulmonary aspiration of gastric contents is the single most common cause of death from anesthesia events. ${ }^{4}$ Similar findings are reported in a French national survey of anesthesia-related mortality. ${ }^{3}$ Furthermore, although effective preventive measures exist, inadequate risk assessment leading to inappropriate airway management is the likely root cause in many cases of aspiration. ${ }^{4,19} \mathrm{~A}$ state-wide audit from Maryland on over 300,000 anesthetics suggests that the incidence may be higher than commonly thought (up to $1 \%$ of all surgical procedures) and that aspiration results in an eightfold increase in in-hospital mortality. ${ }^{19,20}$ Serious morbidity, such as pneumonia, acute respiratory distress syndrome, multiple organ dysfunction, brain damage, and disability are common among survivors. ${ }^{19,20}$ The significance of pulmonary aspiration for patient safety is underscored by its inclusion as one of only three anesthesia-related items in the World Health Organization Surgical Safety Checklist (along with the risk of difficult airway management and equipment/pulse oximeter check). ${ }^{21}$

Point-of-care gastric ultrasound is a valid and reliable tool to define aspiration risk at the bedside prior to induction of anesthesia. ${ }^{11-14,16,22}$ Bedside ultrasound examination provides information about the type of gastric content (empty, clear fluids vs solids) (Fig. 1) and its volume, thus suggesting the level of aspiration risk (Fig. 2). ${ }^{12,13,18}$ In a prospective study of 80 patients undergoing urgent tracheal intubation in an intensive care unit, Koenig et al. used bedside ultrasound to determine gastric fullness and decide on the need for gastric decompression prior to airway management. ${ }^{22}$ Surprisingly, in this group of very ill individuals only $24 \%$ were documented to have any gastric content whatsoever. $^{22}$ Similarly, Martin et al. used bedside ultrasound to assess gastric fullness prior to induction of anesthesia in 60 patients with a diagnosis of appendicitis. ${ }^{23}$ Although conventional teaching would suggest that all patients with appendicitis should be considered to have a full stomach and be at a high risk of aspiration, 39\% of patients presented with an empty stomach. ${ }^{23}$ Thus, pointof-care gastric ultrasound affords clinical anesthesiologists the unprecedented opportunity to individualize assessment of aspiration risk and to tailor anesthetic management to the individual situation rather than limit management to the "standard" approach based on generic assumptions. Two 
recent case reports illustrate how gastric ultrasound can guide management in difficult or conflicting clinical scenarios. Tampo et al. confirmed a likely high risk of aspiration in a patient scheduled for open adhesiotomy after gastric sonography revealed a grade 2 antrum despite a reassuring clinical history, a prolonged fasting interval, and nasogastric decompression. ${ }^{24}$ Conversely, Van de Putte reported a case where, despite a shorter fasting interval than recommended, reassuring ultrasound findings consistent with an empty stomach supported the use of a supraglottic airway and titrated opioid induction on an unstable patient with multiple comorbidities presenting for emergency evacuation of a lower limb hematoma. ${ }^{25}$

The findings of the present study may be considered provocative and potentially controversial since 12 patients (32\% of the sample) underwent elective surgery and anesthesia despite shorter fasting times than recommended. To be precise, in five patients, surgery proceeded rather than being cancelled, and in seven patients, surgery proceeded earlier than was anticipated based on history alone (Fig. 3, Appendix, and Table 2). Nevertheless, almost as many patients in this series $(n=9)$ had their surgery cancelled $(n=6)$ or delayed beyond the pre-test plan following sonographic evidence of a "full stomach" $(n=3)$. The intention of this study was not to refute or disprove current practice guidelines, which we consider to be an essential component of safe anesthesia practice. Rather, our results suggest that the addition of point-of-care gastric ultrasound can allow anesthesiologist to tailor risk assessment and guide anesthetic management to the specific individual rather than to rely strictly on generic assumptions based on average gastric emptying times.

Bedside gastric ultrasound meets all the criteria that define point-of-care ultrasound applications. $^{26}$ It is performed at the patient's bedside; it aims to answer a well-defined diagnostic question intended to guide clinical care; it is focused and goal-directed, and the findings are easily recognizable. ${ }^{16}$ In addition, it is easily learned ${ }^{27}$ and quickly performed. ${ }^{22}$ The fact that a second ultrasound exam was requested by the attending anesthesiologists in 23 subjects suggests that in many cases ultrasound was used as a "monitor" to evaluate gastric content (a dynamic situation) over time before a final management decision was taken.

Strengths of our study include its generalizability as patients were scheduled for various surgical procedures requiring different anesthetic techniques. Seventeen staff anesthesiologists of both sexes with a wide range in age and years in practice referred patients to the study and made patient care decisions. This possibly reflects a range of individual practice patterns and risk tolerance. Five different anesthesiologists performed the ultrasound assessments. The scanning protocol and the reporting method used have been well described and validated and can be readily learned and applied. $^{11,12,14,27}$ The post-test patient management strategy represents "true" patient management and not "ideal" patient management based on an algorithm. The attending anesthesiologists (and not study investigators) had full discretion and control over patient care decisions and were not "bound" by the test result or a strict algorithm.

Our study has several limitations. First, unlike real-life point-of-care ultrasound use, the anesthesiologists performing the scan were not the same physicians making patient care decisions. Nevertheless, this was a conscious decision by the authors to minimize the bias in patient care that could affect study outcomes. The limited familiarity of the attending anesthesiologists' staff with this new diagnostic tool is a possible limitation of the study. Conceivably, individual staff anesthesiologists may have accepted management decisions with a view that they were doing so with implicit support from a colleague performing the ultrasound test rather than based on a full and in-depth understanding of the implications of the ultrasound findings. In addition, the study included non-pregnant adults with a body mass index $<40 \mathrm{~kg} \cdot \mathrm{m}^{-2}$. Caution should be used when extrapolating these findings to other patient populations not included in the study and in whom gastric ultrasound has not yet been fully validated. Finally, although all patients in the study underwent a safe anesthetic with no episodes of regurgitation or aspiration, our study is too small to draw any definitive conclusions regarding the impact of gastric sonography on overall patient safety given the relatively low baseline incidence of pulmonary aspiration.

In summary, this prospective case series suggests that a standardized point-of care gastric ultrasound examination informs anesthesiologists' perceived level of aspiration risk and leads to changes in anesthetic management in a significant proportion of elective patients who did not follow fasting instructions.

Conflicts of interest Dr. Vincent Chan is a member of the Medical Advisory Board for Smiths Medical and Philips. He received an honorarium from AbbVie in the past year and equipment support for research from Ultrasonix.

Funding and support Drs Niazi, Chin, and Perlas received support for academic time from the Department of Anesthesia, University of Toronto through a Merit Award Competition, 2013-2015, 2013-2015, and 2011-2013, respectively.

\section{References}

1. Warner MA, Warner ME, Weber JG. Clinical significance of pulmonary aspiration during the perioperative period. Anesthesiology 1993; 78: 56-62.

2. Shime N, Ono A, Chihara E, Tanaka Y. Current status of pulmonary aspiration associated with general anesthesia: a nationwide survey in Japan (Japanese). Masui 2005; 54: 1177-85. 
3. Lienhart A, Auroy $Y$, Pequignot $F$, et al. Survey of anesthesiarelated mortality in France. Anesthesiology 2006; 105: 1087-97.

4. Cook TM, Woodall N, Frerk C, Fourth National Audit Project. Major complications of airway management in the UK: results of the Fourth National Audit Project of the Royal College of Anaesthetists and the Difficult Airway Society. Part 1: anaesthesia. Br J Anaesth 2011; 106: 617-31.

5. American Society of Anesthesiologists Committee. Practice guidelines for pre-operative fasting and the use of pharmacologic agents to reduce the risk of pulmonary aspiration: application to healthy patients undergoing elective procedures: an updated report by the American Society of Anesthesiology Committee on Standards and Practice Parameters. Anesthesiology 2011; 114: 495-511.

6. Merchant $R$, Chartrand D, Dain S, et al. Guidelines to the practice of anesthesia. Revised edition 2015. Can J Anesth 2015; 62: 54-67.

7. Darwiche $G$, Bjorgell O, Thorsson O, Almer LO. Correlation between simultaneous scintigraphic and ultrasonographic measurement of gastric emptying in patients with type 1 diabetes mellitus. J Ultrasound Med 2003; 22: 459-66.

8. De Schoenmakere $G$, Vanholder R, Rottey $S$, Duym P, Lameire $N$. Relationship between gastric emptying and clinical and biochemical factors in chronic haemodialysis patients. Nephrol Dial Transplant 2001; 16: 1850-5.

9. Ishizu H, Shiomi S, Kawamura E, et al. Gastric emptying in patients with chronic liver diseases. Ann Nucl Med 2002; 16: 177-82.

10. Splinter WM. From the Journal archives: Gastric fluid volume and $\mathrm{pH}$ in elective patients following unrestricted oral fluid until three hours before surgery. Can J Anesth 2014; 61: 1126-9.

11. Perlas A, Chan VW, Lupu CM, Mitsakakis N, Hanbidge A. Ultrasound assessment of gastric content and volume. Anesthesiology 2009; 111: 82-9.

12. Perlas A, Mitsakakis N, Lui L, et al. Validation of a mathematical model for ultrasound assessment of gastric volume by gastroscopic examination. Anesthesiology 2013; 116: 357-63.

13. Bouvet L, Mazoit JX, Chassard D, Allaouchiche B, Boselli E, Benhamou D. Clinical assessment of the ultrasonographic measurement of antral area for estimating preoperative gastric content and volume. Anesthesiology 2011; 114: 1086-92.

14. Kruisselbrink $R$, Arzola C, Endersby $R$, Tse C, Chan V, Perlas A. Intra- and interrater reliability of ultrasound assessment of gastric volume. Anesthesiology 2014; 121: 46-51.
15. Perlas A, Davis L, Khan M, Mitsakakis N, Chan VW. Gastric sonography in the fasted surgical patient: a prospective descriptive study. Anesth Analg 2011; 113: 93-7.

16. Cubillos J, Tse C, Chan VW, Perlas A. Bedside ultrasound assessment of gastric content: an observational study. Can J Anesth 2012; 59: 416-23.

17. Von Elm E, Altman DG, Egger $M$, et al. The Strengthening the Reporting of Observational Studies in Epidemiology (STROBE) statement: guideline for reporting observational studies. J Clin Epidemiol 2008; 61: 344-9.

18. Van de Putte P, Perlas A. Ultrasound assessment of gastric content and volume. Br J Anaesth 2014; 113: 12-22.

19. Sakai T, Planinsic RM, Quinlan JJ, Handley LJ, Kim TJ, Hilmi $I A$. The incidence and outcomes of perioperative pulmonary aspiration in a university hospital: a 4-year retrospective analysis. Anesth Analg 2006; 103: 941-7.

20. Kozlow JH, Berenholtz SM, Garrett E, Dorman T, Provonost PJ. Epidemiology and impact of aspiration pneumonia in patients undergoing surgery in Maryland, 1999-2000. Crit Care Med 2003; 31: 1930-7.

21. World Health Organization. WHO Surgical Safety Checklist. Available from URL: www.who.int/patientsafety/safesurgery/ checklist/en (accessed June 2015).

22. Koenig SJ, Lakticova $V$, Mayo PH. Utility of ultrasonography for detection of gastric fluid during urgent endotracheal intubation. Intensive Care Med 2011; 37: 627-31.

23. Martin $R$, Plante $F$. Stomach content and ultrasound technology in appendicitis. J Clin Anesth 2008; 20: 485.

24. Tampo A, Suzuki A, Ijiri E, Kunisawa T, Iwasaki H. Preanesthetic gastric assessment with sonography for a patient with a full stomach. J Clin Anesth 2013; 25: 164-5.

25. Van de Putte $P$. Bedside gastric ultrasonography to guide anesthetic management in a nonfasted emergency patient. J Clin Anesth 2013; 25: 165-6.

26. Kendall JL, Hoffenberg SR, Smith RS. History of emergency and critical care ultrasound: the evolution of a new imaging paradigm. Crit Care Med 2007; 35(5 Suppl): S126-30.

27. Arzola C, Carvalho J, Cubillos J, Ye XY, Perlas A. Anesthesiologists' learning curves for bedside qualitative ultrasound assessment of gastric content: a cohort study. Can J Anesth 2013; 60: 771-9. 